\title{
Leader-follower Consensus of Unicycles with Communication Range Constraints via Smooth Time-invariant Feedback
}

\author{
Esteban Restrepo Antonio Loría Ioannis Sarras Julien Marzat
}

\begin{abstract}
We solve the full-consensus problem (in position and orientation) with connectivity maintenance for multiple nonholonomic vehicles in a leader-follower configuration. We rely on a polar-coordinates based model which is more natural for the problem setting. The proposed control law is smooth (in the domain of definition) time-invariant and uses only relative measurements, making it more suited for implementation. We establish asymptotic convergence to the consensus manifold as well as connectivity maintenance using the Lyapunov's first method and cascaded systems theory. In addition, we illustrate our theoretical contributions experimentally.
\end{abstract}

Index Terms-Formation control, persistency of excitation, Lyapunov design, nonholonomic systems

\section{INTRODUCTION}

$\mathbf{I}$ $\mathrm{N}$ the literature on consensus of nonholonomic vehicles two main problems are addressed, position consensus, in which case all agents are to converge to the same position with arbitrary or predetermined orientation, and full consensus, in which case, agreement on the orientation is also sought.

In either case, most often a Cartesian-coordinates-based model is used, for which the origin is not stabilisable via smooth invariant feedback. For instance, the controllers proposed in [1], [2] are time-varying and they guarantee position consensus. Time-varying feedback is also used in [3]-[5], but for full-consensus-based formation control. In [6], on the other hand, a time-invariant non-smooth feedback positionconsensus controller is reported.

Now, in these, as in many other works, it is assumed that graphs are undirected and that the communication graph is connected at all times. Hence, each agent has permanent access to its neighbours' data, either by transmission or by sensing. In applications of multi-agent systems, however, the communication or sensing devices embarked on autonomous vehicles often have limited range, that is, each agent can only communicate with or sense another agent if it is within a limited distance from the vehicle's position. Therefore, assuming the existence of a connected graph at all times representing the information exchange, although necessary from a theoretical viewpoint, turns out to be conservative from a practical one.

E. Restrepo, I. Sarras, and J. Marzat are with DTIS, ONERA, Université Paris-Saclay, F-91123 Palaiseau, France. E-mail: \{esteban.restrepo, ioannis.sarras, julien.marzat $\}$ @onera.fr. A. Loría is with CNRS, 91192 Gifsur-Yvette, France. E-mail: antonio.loria@centralesupelec.fr E. Restrepo and A. Loría are also with L2S-CentraleSupélec, Université Paris-Saclay, Saclay, France. . The work of A. Loría was supported by the French ANR via project HANDY, contract number ANR-18-CE40-0010 and by CEFIPRA under the grant number 6001-A.
Therefore, much effort has been spent recently on the design of connectivity maintenance strategies for nonholonomic systems. However, most works in the literature address only the problem of position consensus. Moreover, the consensus protocols used are, for the most part, either non-smooth or time-varying. Besides increasing the complexity of the analysis (use of non-smooth Lyapunov analysis, non-autonomous systems theory, persistency of excitation, etc.), they may give way to undesirable behavior, such as oscillations. In [7] and [8] a navigation-function-based time-varying controller with connectivity maintenance is proposed, respectively, for undirected and directed graphs; nevertheless, it presents some problems inherent to the navigation-function framework such as local minima and the need to have a bounded workspace. In [9] and [10] connectivity-preserving controllers are proposed, respectively, for undirected and leader-follower topologies based on barrier functions. However, only position consensus is addressed. In [11], position consensus-based formation with connectivity maintenance is achieved using barrier functions but assuming a complete undirected graph, that is, assuming that each agent has access to the information of every other agent in the system at all times, which can prove limiting in realistic practical scenarios. In [12], a discontinuous controller is presented to guarantee connectivity, but consensus is not considered and global parameters must be estimated, thereby increasing the complexity of the controller. Estimation of global parameters is also used in [13] to achieve practical stability of a desired formation, but only position consensus is addressed.

In this paper we present a distributed controller for full consensus (in position and orientation) of nonholonomic vehicles in a leader-follower configuration, under relative distance constraints. More precisely, the full-consensus algorithm proposed in [14] is extended herein to address, also, the problem of connectivity maintenance. Remarkably, the proposed control law is smooth (in the domain of definition) time-invariant. Moreover, it has the advantage of relying only on relative measurements of distance-to-follower and line-of-sight angles, which makes our controller more suitable for implementation and practical use. The latter is made possible by employing a polar-coordinates model based on the one proposed in [15]. We also remark that the leader-follower configuration of the robots means that the graph topology corresponds to that of a (minimal) directed spanning tree.

In Section II are presented the model and the problem statement. The main results are presented in Section III and 
are illustrated via experimental results in Section IV. Some concluding remarks are given in Section V.

\section{Model and Problem Statement}

Notation. The real $n$-coordinate space, with $n \in \mathbb{N}$, is denoted as $\mathbb{R}^{n} ; \mathbb{R}_{>0}^{n}$ and $\mathbb{R}_{>0}^{n}$ are the sets of real $n$-vectors with all elements non-negative and positive, respectively. The notation $\|x\|$ is used for the Euclidean norm of a vector $x \in \mathbb{R}^{n}$. A function $\gamma: \mathbb{R}_{\geq 0} \rightarrow \mathbb{R}_{\geq 0}$ is said to be of class $\mathcal{K}(\gamma \in$ $\mathcal{K})$, if it is continuous, strictly increasing and zero at zero. If moreover $\gamma(s) \rightarrow \infty$ as $s \rightarrow \infty$, we say that $\gamma \in \mathcal{K}_{\infty}$. A digraph, denoted $\mathcal{G}=(\mathcal{V}, \mathcal{E})$, is defined by a set of nodes, $\mathcal{V}:=\{1,2, \ldots, n\}$ whose elements correspond to the labels of the agents' states and a set of edges, $\mathcal{E} \subseteq \mathcal{V}^{2}$ of cardinality $m$, that represents the communication between a pair of nodes. A directed edge $e_{k}$, with $k \leq m$, is an ordered pair $(i, j) \in \mathcal{E}$ if and only if a connection exists from node $i$ to node $j$. The distance $d(i, j)$ between nodes $i, j \in \mathcal{V}$ is the number of edges in the shortest path from $i$ to $j$. A directed tree is a subgraph consisting in a root node, with no parent, and a set of nodes reachable from the root. A directed spanning tree $\mathcal{G}_{\mathcal{T}} \subset \mathcal{G}$ is a directed tree containing all the nodes in $\mathcal{G}$.

Unicycle systems are typically modelled by the equations

$$
\begin{aligned}
& \dot{x}_{j}=v_{j} \cos \theta_{j} \\
& \dot{y}_{j}=v_{j} \sin \theta_{j} \\
& \dot{\theta}_{j}=\omega_{j}, \quad j \leq n
\end{aligned}
$$

where $q_{j}=\left[x_{j}, y_{j}\right]^{\top} \in \mathbb{R}^{2}$ is the vector of Cartesian coordinates, $\theta_{j} \in(-\pi, \pi]$ denotes the vehicle's orientation with respect to the axis of the abscissae, and the control inputs are the linear speed, $v_{j} \in \mathbb{R}$, and the angular velocity, $\omega_{j} \in \mathbb{R}$. One of the best known characteristics of nonholonomic systems (1) is that, in general, equilibria in the space of $(x, y, \theta)$ are not reachable via smooth time-invariant feedback. In [16] necessary conditions for stabilisability of the positionconsensus manifold (i.e., disregarding orientations) via smooth invariant feedback are given.

In [15] an alternative, polar-coordinates-based, model for unicycles is proposed. Such model is not defined at the origin (zero distance to the target point), but equilibria are reachable via smooth time-invariant feedback. Furthermore, used in a leader-follower configuration, this model has the additional advantage of naturally leading to the design of controllers that rely only on local relative measurements.

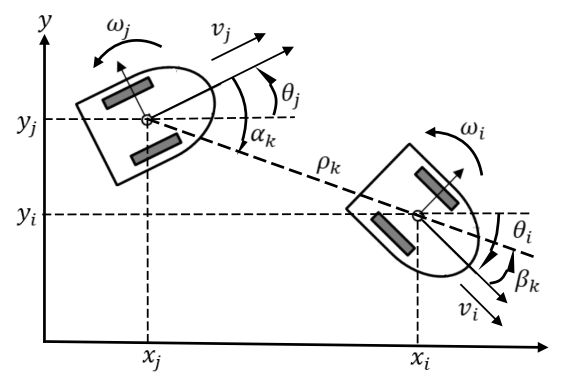

Fig. 1. Leader-follower scheme and polar-coordinates variables
After [15], for every pair of leader and follower vehicles, labelled $i$ and $j$ respectively, let $\rho_{k}$ denote the distance separating them, let $\beta_{k}$ denote the angle between the line of sight and the leader's direction of motion, and let $\alpha_{k}$ denote the angle between the line of sight and the follower's direction of motion - see Figure 1. That is,

$$
\begin{aligned}
\rho_{k} & :=\left\|q_{i}-q_{j}\right\| \\
\beta_{k} & :=\arctan \left(\frac{y_{i}-y_{j}}{x_{i}-x_{j}}\right)-\theta_{i}, \quad \forall \rho_{k}>0 \\
\alpha_{k} & :=\arctan \left(\frac{y_{i}-y_{j}}{x_{i}-x_{j}}\right)-\theta_{j}, \quad \forall \rho_{k}>0
\end{aligned}
$$

Note that the three-dimensional space of $\left(x_{j}, y_{j}, \theta_{j}\right)$ is mapped into another space of dimension 3 , corresponding to the relative coordinates $\left(\rho_{k}, \beta_{k}, \alpha_{k}\right) \in \mathbb{R}_{\geq 0} \times(-\pi, \pi]^{2}$. Indeed, $\rho_{k}=0$ and $\beta_{k}=\alpha_{k}$ is equivalent to $q_{i}=q_{j}$ and $\theta_{i}=\theta_{j}$. Therefore, the solutions to Eqs. (3) correspond to the leader-follower relative error trajectories for the pair of index $k$ and the full consensus control goal is reached if $\left(\rho_{k}, \beta_{k}, \alpha_{k}\right) \rightarrow(0,0,0)$ asymptotically for all $k \leq m$, where $m$ corresponds to the number of pairs of leaders-followers.

Then, from a control viewpoint, a network of agents interacting with each other in a leader-follower configuration, corresponds to $m$ interconnected dynamical systems,

$$
\begin{aligned}
& \dot{\rho}_{k}=v_{i} \cos \beta_{k}-v_{j} \cos \alpha_{k} \\
& \dot{\beta}_{k}=\frac{1}{\rho_{k}}\left[-v_{i} \sin \beta_{k}+v_{j} \sin \alpha_{k}\right]-\omega_{i} \\
& \dot{\alpha}_{k}=\frac{1}{\rho_{k}}\left[-v_{i} \sin \beta_{k}+v_{j} \sin \alpha_{k}\right]-\omega_{j},
\end{aligned}
$$

which result from differentiating on both sides of (2) with respect to time and using (1). In the latter equations $v_{j}$ and $\omega_{j}$ are the control inputs, and $v_{i}$ and $\omega_{i}$, which are respectively the leader's velocity and angular rate, are considered as external signals.

For the purpose of analysis, the interconnections are represented using a generic spanning-tree graph in which a triple $\left(\rho_{k}, \beta_{k}, \alpha_{k}\right)$ corresponds to the state of an edge $e_{k}$ in the tree -see Figure 2. A sequence of one-leader-one-follower pairs forms a directed path and the overall dynamical system has a convenient cascaded structure [17], in which the dynamics of each subsystem corresponds to (3). As we show farther below, the cascaded structure is conserved for general spanning-tree topologies; this is fundamental to our control approach.

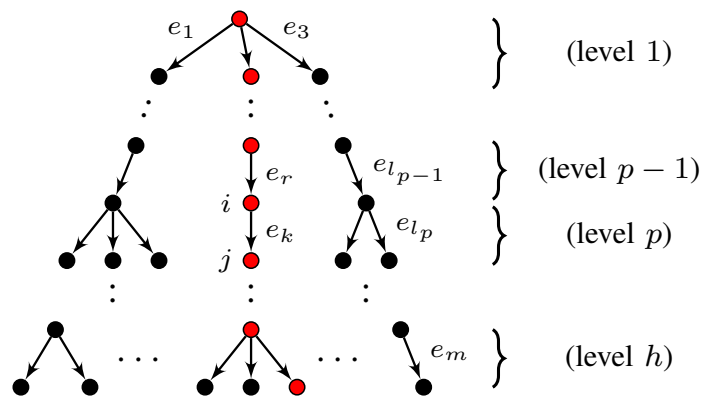

Fig. 2. Directed spanning tree $\mathcal{G}_{\mathcal{T}}$. Open directed chain illustrated in red 
Now, while the existence of a directed spanning tree is necessary for consensus, taking this for granted may be conservative in certain applications. In this paper, we develop upon the preliminary results obtained in [14] to design controllers that ensure the following property.

Definition 1 (Connectivity maintenance): For each $k \leq m$, let $\Delta_{k}>0$ denote the maximal distance between the leaderfollower pair $i$ and $j$ such that the communication between them, through the arc $e_{k}=(i, j)$, is reliable. We say that the graphs connectivity is maintained if the set

$$
\mathcal{J}:=\left\{\rho_{k} \in \mathbb{R}_{\geq 0}: \rho_{k}<\Delta_{k}, \forall k \leq m\right\},
$$

where $\rho_{k}$ is defined in (2a), is forward invariant. That is, if $\rho_{k}(0)<\Delta_{k}$ implies that $\rho_{k}(t) \in \mathcal{J}$ for all $t \geq 0$.

The design of controllers that guarantee connectivity maintenance relies on so-called barrier Lyapunov functions. In turn, the latter are defined as the anti-derivative of so-called connectivity potential functions.

Definition 2 (Connectivity potential): Let $p_{0} \in \mathbb{R}$ and, for each $k \leq m$, let $\mathcal{B}_{\Delta_{k}}:=\left\{\rho_{k} \in \mathbb{R}_{\geq 0}: \rho_{k}<\Delta_{k}\right\}$. Let $\phi_{k}:\left[0, \Delta_{k}^{2}\right) \rightarrow \mathbb{R}_{\geq 0}, s \mapsto \phi_{k}(s)$, be $\overline{\mathcal{C}}^{1}$ and non-decreasing on $\left[0, \Delta_{k}^{2}\right)$, such that $\phi_{k}(s) \rightarrow \infty$ as $s \rightarrow \Delta_{k}^{2}$, and the connectivity potential $p_{k}: \mathcal{B}_{\Delta_{k}} \rightarrow \mathbb{R}_{>0}$, defined as

$$
p_{k}\left(\rho_{k}\right):=\frac{\partial \phi_{k}}{\partial s}\left(\rho_{k}^{2}\right)
$$

is also non-decreasing, $p_{k}\left(\rho_{k}\right) \geq p_{0}>0$ for all $\rho_{k}<\Delta_{k}$, and $p_{k}\left(\rho_{k}\right) \rightarrow \infty$ as $\rho_{k} \rightarrow \Delta_{k}$.

Then, we define a barrier function ${ }^{1} U_{k}: \mathcal{B}_{\Delta_{k}} \rightarrow \mathbb{R}_{\geq 0}$ as

$$
U_{k}\left(\rho_{k}\right):=\phi_{k}\left(\rho_{k}^{2}\right)
$$

and it satisfies

$$
\frac{\partial U_{k}}{\partial \rho_{k}}=2 p_{k}\left(\rho_{k}\right) \rho_{k}
$$

Thus, the control problem is to define, for each $j \leq n$, control laws $v_{j}$ and $\omega_{j}$ such that, for the system (3), $\left(\rho_{k}, \beta_{k}, \alpha_{k}\right) \rightarrow(0,0,0)$ asymptotically for all $k \leq m$ and that connectivity, according to Definition 1 , is maintained for all initial conditions such that $\rho_{k}(0) \in \mathcal{J}$ and for all $t \geq 0$.

\section{MAIN RESULTS}

\section{A. Control approach}

Before presenting the control laws that solve the problem of full consensus with connectivity maintenance, we first introduce the rationale used for their design. Exploiting the natural cascaded structure of the system, we follow a backstepping procedure. To see this more clearly, consider first the system (3) for a single edge $e_{k}$ with zero leader velocities, that is, $v_{i}=\omega_{i}=0$. It is clear from (3) that the angular rate $\omega_{j}$ directly affects only the dynamics of $\alpha_{k}$, whereas the evolution of $\rho_{k}$ and $\beta_{k}$ are determined by $\alpha_{k}$. Hence, the first step is to design the actual control input $v_{j}$ and a virtual control input $\alpha_{k}^{*}: \mathbb{R}_{\geq 0} \times \mathbb{R} \rightarrow(-\pi / 2, \pi / 2) ;\left(\rho_{k}, \beta_{k}\right) \mapsto \alpha_{k}^{*}\left(\rho_{k}, \beta_{k}\right)$ such

\footnotetext{
${ }^{1}$ Examples of functions satisfying the properties (6)-(7) and Definition 2 are the logarithmic barrier Lyapunov functions proposed in [18].
}

that $\alpha_{k}^{*}(0,0)=0$, in order to make $\rho_{k}(t) \rightarrow 0$ and $\beta_{k}(t) \rightarrow 0$ asymptotically. Defining the virtual input

$$
\alpha_{k}^{*}:=\arctan \left(-c_{3} \beta_{k}\right), \quad c_{3}>0,
$$

and using the identities

$\sin (\arctan (s))=\frac{s}{\sqrt{1+s^{2}}}, \quad \cos (\arctan (s))=\frac{1}{\sqrt{1+s^{2}}}$,

we obtain a dynamical subsystem of the form

$$
\dot{\rho}_{k}=-\frac{v_{j}}{\sqrt{1+\left(c_{3} \beta_{k}\right)^{2}}}, \quad \dot{\beta}_{k}=-c_{3} \frac{v_{j}}{\rho_{k} \sqrt{1+\left(c_{3} \beta_{k}\right)^{2}}} \beta_{k} .
$$

Note that the latter can be rendered asymptotically stable by taking the input $v_{j}$ proportional to $\rho_{k}$.

Next, defining the error variable $\tilde{\alpha}_{k}:=\alpha_{k}-\alpha_{k}^{*}$, we obtain

$$
\begin{aligned}
& \dot{\rho}_{k}=-\frac{v_{j}}{\sqrt{1+\left(c_{3} \beta_{k}\right)^{2}}}-v_{j}\left[\cos \left(\alpha_{k}\right)-\cos \left(\alpha_{k}^{*}\right)\right] \\
& \dot{\beta}_{k}=-c_{3} \frac{v_{j}}{\rho_{k} \sqrt{1+\left(c_{3} \beta_{k}\right)^{2}}} \beta_{k}+v_{j}\left[\sin \left(\alpha_{k}\right)-\sin \left(\alpha_{k}^{*}\right)\right] \\
& \dot{\tilde{\alpha}}_{k}=\frac{v_{j}}{\rho_{k}} \sin \alpha_{k}-\dot{\alpha}_{k}^{*}-\omega_{j}
\end{aligned}
$$

so, following a backstepping procedure, the angular rate $\omega_{j}$ is designed so that $\tilde{\alpha}_{k} \rightarrow 0$, or equivalently, $\alpha_{k} \rightarrow \alpha_{k}^{*}$.

The previous control design is effective in stabilizing one robot to a point without considering proximity constraints. For the purpose of leader-follower consensus of multi-agent systems (3) we follow the previous ideas and, to satisfy the connectivity-maintenance requirement, the input $v_{j}$ is not simply chosen proportional to $\rho_{k}$, but as a function of the gradient of a barrier function $W_{k}: \mathcal{B}_{\Delta_{k}} \rightarrow \mathbb{R}_{\geq 0}$ which encodes the distance constraints. This function is defined as

$$
W_{k}\left(\rho_{k}\right):=\frac{1}{2} \rho_{k}^{2}+\frac{\kappa_{\rho}}{2} U_{k}\left(\rho_{k}\right),
$$

where $\kappa_{\rho}>0$ is a design parameter and $U_{k}$ is as in (6).

Then, denoting the gradient of $W_{k}$ as $\partial_{\rho} W_{k}:=\frac{\partial W_{k}}{\partial_{\rho_{k}}}$, we introduce the distributed control laws that solve the full consensus problem with connectivity maintenance as:

$$
\begin{aligned}
& v_{j}:=c_{1} \sum_{k \leq m} a_{j k} \eta_{k} \partial_{\rho} W_{k}\left(\rho_{k}\right), \quad \eta_{k}:=\sqrt{1+\left(c_{3} \beta_{k}\right)^{2}} \\
& \omega_{j}:=\sum_{k \leq m} a_{j k}\left[c_{2} \tilde{\alpha}_{k}+\left[\psi_{k}+\left[1+\frac{c_{3}}{\eta_{k}^{2}}\right] \frac{\sin \left(\alpha_{k}\right)}{\rho_{k}}\right] \sum_{i \leq n} a_{i k} v_{i}\right]
\end{aligned}
$$

where $c_{1}, c_{2}>0$ are design constants, $\alpha_{k}^{*}$ is defined in (8),

$$
\psi_{k}:=-\partial_{\rho} W_{k} \frac{\left[\cos \left(\alpha_{k}\right)-\cos \left(\alpha_{k}^{*}\right)\right]}{\tilde{\alpha}_{k}}+\frac{\beta_{k}}{\rho_{k}} \frac{\left[\sin \left(\alpha_{k}\right)-\sin \left(\alpha_{k}^{*}\right)\right]}{\tilde{\alpha}_{k}},
$$

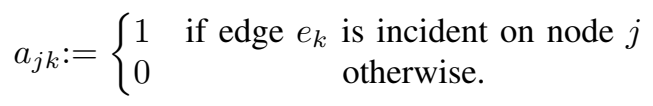




\section{B. The multi-agent system in closed loop}

So far, we considered the leader-follower pair (3) as an individual system. Consider, now a multi-agent system composed of $n$ unicycles communicating through an arbitrary directed spanning tree. Each robot has only one leader, but may have several followers, so the tree has $h \leq n-1$ levels based on the distance to the root. Now, let $\mathcal{E}_{p} \subset \mathcal{E}$ denote the set of edges such that the distance from its terminal node to the root of the tree, labelled "1", is equal to $p \leq h$, i.e., $\mathcal{E}_{p}:=\left\{e_{k}=(i, j) \in \mathcal{E}: d(1, j)=p\right\}$. Without loss of generality, assume that each level $p$ of the tree contains an $l_{p}$ number of edges, such that $1 \leq l_{p} \leq m, \sum_{p=1}^{h} l_{p}=m$ - see Figure 2. Then, we define a multi-variable model containing the three states of all the edges belonging to a level of the tree. To that end, for each level $p$ having $l_{p}$ arcs labelled $e_{k}$ with $k \in\left[l_{p-1}+1, l_{p}\right]$ we define the closed-loop state variables

$$
\xi_{p}:=\left[\begin{array}{lll}
\xi_{p, 1}^{\top} & \cdots & \xi_{p, l_{p}}^{\top}
\end{array}\right]^{\top}, \quad \xi_{p, k}:=\left[\begin{array}{lll}
\rho_{k} & \beta_{k} & \tilde{\alpha}_{k}
\end{array}\right]^{\top} .
$$

Note that the dimension of $\xi_{p}$ is $3 l_{p}$, that is, it depends on the number of arcs in the tree level.

Using this notation, the systems (3) in closed loop with (11), with $k \leq m$ and for the considered graph, can be written in the compact cascaded-system form,

$$
\left\{\begin{aligned}
\dot{\xi}_{h} & =f_{h}\left(\xi_{h}\right)+g_{h}\left(\xi_{h}, \xi_{h-1}\right), \\
& \vdots \\
\dot{\xi}_{p} & =f_{p}\left(\xi_{p}\right)+g_{p}\left(\xi_{p}, \xi_{p-1}\right) \\
& \vdots \\
\dot{\xi}_{2} & =f_{2}\left(\xi_{2}\right)+g_{2}\left(\xi_{2}, \xi_{1}\right) \\
\dot{\xi}_{1} & =f_{1}\left(\xi_{1}\right)
\end{aligned}\right.
$$

where, for each $p \leq h$,

$$
\begin{aligned}
f_{p}\left(\xi_{p}\right) & :=\left[f_{p, 1}\left(\xi_{p, 1}\right)^{\top} \ldots f_{p, l_{p}}\left(\xi_{p, l_{p}}\right)^{\top}\right]^{\top}, \\
g_{p}\left(\xi_{p}, \xi_{p-1}\right) & :=\left[g_{p, 1}\left(\xi_{p, 1}, \xi_{p-1}\right)^{\top} \ldots g_{p, l_{p}}\left(\xi_{p, l_{p}}, \xi_{p-1}\right)^{\top}\right]^{\top},
\end{aligned}
$$

and the nominal system, $\dot{\xi}_{p, k}=f_{p, k}\left(\xi_{p, k}\right)$, corresponds to

$$
\begin{aligned}
& \dot{\rho}_{k}=-c_{1} \partial_{\rho} W_{k}\left[1+\left[\cos \left(\alpha_{k}\right)-\cos \left(\alpha_{k}^{*}\right)\right] \eta_{k}\right] \\
& \dot{\beta}_{k}=-\frac{c_{1}}{\rho_{k}} \partial_{\rho} W_{k}\left[c_{3} \beta_{k}-\left[\sin \left(\alpha_{k}\right)-\sin \left(\alpha_{k}^{*}\right)\right] \eta_{k}\right] \\
& \dot{\tilde{\alpha}}_{k}=-c_{2} \tilde{\alpha}_{k}-c_{1} \psi_{k} \eta_{k} \partial_{\rho} W_{k}
\end{aligned}
$$

where we recall that $\alpha_{k}=\tilde{\alpha}_{k}+\alpha_{k}^{*}$.

The interconnection term $g_{p}\left(\xi_{p}, \xi_{p-1}\right)$ depends on states relative to the $p$-th level in the tree and to the previous one in the following way. Fix $k \in\left[l_{p-1}+1, l_{p}\right]$ and $r \in\left[l_{p-2}+1, l_{p-1}\right]$ in a manner that the edge $e_{r} \in \mathcal{E}_{p-1}$ is incident on $e_{k} \in \mathcal{E}_{p}$, that is, so that the terminal node of $e_{r}$ is the initial node of $e_{k}$ —see Figure 2. Let $\xi_{p-1, r}:=\left[\begin{array}{lll}\rho_{r} & \beta_{r} & \tilde{\alpha}_{r}\end{array}\right]^{\top}$ be the state associated to $e_{r}$. Then,

$$
g_{p, k}\left(\xi_{p, k}, \xi_{p-1}\right)=\left[\begin{array}{c}
c_{1} \cos \left(\beta_{k}\right) \eta_{r} \partial_{\rho} W_{r} \\
\tilde{g}_{\beta}\left(\xi_{p, k}, \xi_{p-1, r}\right) \\
\tilde{g}_{\alpha}\left(\xi_{p, k}, \xi_{p-1, r}\right)
\end{array}\right],
$$

where $\eta_{r}:=\sqrt{1+\left(c_{3} \beta_{r}\right)^{2}}$,

$\tilde{g}_{\beta}:=-c_{1} \eta_{r} \partial_{\rho} W_{r}\left[\frac{\sin \left(\beta_{k}\right)}{\rho_{k}}+\left[1+\frac{c_{3}}{\eta_{r}^{2}}\right] \frac{\sin \left(\alpha_{r}\right)}{\rho_{r}}+\psi_{r}\right]-c_{2} \tilde{\alpha}_{r}$ and

$$
\begin{array}{r}
\tilde{g}_{\alpha}:=-\frac{c_{2} c_{3}}{\eta_{k}^{2}} \tilde{\alpha}_{r}-c_{1} \eta_{r} \partial_{\rho} W_{r}\left[\frac{c_{3}}{\eta_{k}^{2}}\left[\psi_{r}+\left[1+\frac{c_{3}}{\eta_{r}^{2}}\right] \frac{\sin \left(\alpha_{r}\right)}{\rho_{r}}\right]\right. \\
\left.+\left[1+\frac{c_{3}}{\eta_{k}^{2}}\right] \frac{\sin \left(\beta_{k}\right)}{\rho_{k}}\right]
\end{array}
$$

where $\alpha_{r}=\tilde{\alpha}_{r}+\alpha_{r}^{*}$.

We stress that $\partial_{\rho} W_{r}$ is a function of $\rho_{r}$ only and $\left[\partial_{\rho} W_{r}\right](0)=0$. Therefore, from the definition of $g_{p}$ we have $g_{p}\left(\xi_{p}, 0\right) \equiv 0$. Hence, based on cascaded-systems theory [19], one can assert that if for every $p \in[2, h+1]$ the solution of $\dot{\xi}_{p-1}=f_{p-1}\left(\xi_{p-1}\right)$ converges to zero and if, for every $p \in[2, h]$, the solutions of $\dot{\xi}_{p}=f_{p}\left(\xi_{p}\right)+g_{p}\left(\xi_{p}, \xi_{p-1}\right)$, denoted $\xi_{p}(t)$, remain bounded, we also have $\xi_{p}(t) \rightarrow 0$. This is established in our main statement, which is presented below.

\section{Main statement}

Proposition 1 (Main result): Consider $n$ unicycle agents described by (1) and communicating over a directed spanning tree $\mathcal{G}_{\mathcal{T}}(\mathcal{V}, \mathcal{E})$. The smooth time-invariant controller (11)-(13) achieves full consensus with connectivity maintenance, i.e., $q_{i} \rightarrow q_{j}, \theta_{i} \rightarrow \theta_{j}$ and $\rho_{k}(t) \in \mathcal{J}$, for all $e_{k}=(i, j) \in \mathcal{E}$, $k \leq m$, and for all initial conditions $\left(\rho_{k}(0), \beta_{k}(0), \alpha_{k}(0)\right)$ such that $\rho_{k}(0) \in \mathcal{J} \backslash\{0\}$ - see Definition 1.

Proof: The proof follows the rationale of [19, Lemma 1]. To that end we establish that for each nominal system, $\dot{\xi}_{p}=f_{p}\left(\xi_{p}\right)$, we have $\xi_{p}(t) \rightarrow 0$ and that the solutions of (15) are bounded. Assume, for the time-being (this assumption will be proven later), that the solutions of (15) are such that $\rho_{k}(t) \in \mathcal{J}$ for all $k \leq m$ and all $t \geq 0$. Let $k \leq m$ and $p \leq h$ be arbitrarily fixed and for the edge $e_{k} \in \mathcal{E}_{p}$, consider the Lyapunov function candidate

$$
V_{p, k}\left(\xi_{p, k}\right)=W_{k}\left(\rho_{k}\right)+\frac{1}{2} \tilde{\alpha}_{k}^{2}+\frac{1}{2} \beta_{k}^{2} .
$$

Its total derivative along the trajectories of (16) - see also (12), satisfies

$$
\dot{V}_{p, k}\left(\xi_{p, k}\right)=-c_{1}\left[\partial_{\rho} W_{k}\right]^{2}-c_{2} \tilde{\alpha}_{k}^{2}-\frac{c_{1} c_{3}\left[\partial_{\rho} W_{k}\right]}{\rho_{k}} \beta_{k}^{2} .
$$

Now, from equations (7), (10), and Definition 2, we have

$$
\dot{V}_{p, k}\left(\xi_{p, k}\right) \leq-c_{1} \rho_{k}^{2}-c_{2} \tilde{\alpha}_{k}^{2}-\tilde{c}_{3} \beta_{k}^{2} \leq-c\left\|\xi_{p, k}\right\|^{2}<0,
$$

where $c:=\min \left\{c_{1}, c_{2}, c_{1} c_{3}\left(1+\kappa_{\rho} p_{0}\right)\right\}$. Next, consider the function

$$
V_{p}\left(\xi_{p}\right)=\sum_{k=1}^{l_{p}} V_{p, k}\left(\xi_{p, k}\right) .
$$

In view of (21), we have

$$
\dot{V}_{p}\left(\xi_{p}\right) \leq-c\left\|\xi_{p}\right\|^{2}<0 .
$$

Since $V_{p}$ is positive definite and $\dot{V}_{p}$ is negative definite on its domain of definition, we conclude that the origin for the nominal system (16) is asymptotically stable at the origin.

Next, we establish boundedness of the solutions of (15). For that purpose, fix $p \leq h$ arbitrarily and consider the $(p-1)$-th and the $p$-th equations of the cascaded system (15). 
In view of (17)-(18), for any $r$ and $k$ as chosen previously (such that the edge $e_{k}$ is incident on $e_{r}$ ), the interconnection terms $g_{p, k}\left(\xi_{p, k}, \xi_{p-1}\right)$ satisfy, component-wise,

$$
g_{p, k}\left(\xi_{p, k}, \xi_{p-1}\right) \leq\left[\max \left\{\begin{array}{c}
\gamma_{\rho}\left(\left\|\xi_{p-1, r}\right\|\right) \\
\left.1, \frac{1}{\rho_{k}}\right\} \gamma_{\beta}\left(\left\|\xi_{p-1, r}\right\|\right) \\
\left.1, \frac{1}{\rho_{k}}\right\} \gamma_{\alpha}\left(\left\|\xi_{p-1, r}\right\|\right)
\end{array}\right]\right.
$$

where $\gamma_{\rho}(s), \gamma_{\beta}(s), \gamma_{\alpha}(s) \in \mathcal{K}_{\infty}$ and we recall that $\xi_{p-1, r}=$ $\left[\begin{array}{lll}\rho_{r} & \beta_{r} & \tilde{\alpha}_{r}\end{array}\right]^{\top}$ is the $r$-th entry of $\xi_{p-1}$. Then, consider the Lyapunov function in (19) with $p=2, k \in\left[l_{1}+1, l_{2}\right]$, and $r \in\left[1, l_{1}\right]$. From (20) and (24), we have

$$
\begin{aligned}
& \dot{V}_{2, k}\left(\xi_{2, k}\right) \leq-c_{1}\left[\partial_{\rho} W_{k}\right]^{2}-c_{2} \tilde{\alpha}_{k}^{2}-\tilde{c}_{3} \beta_{k}^{2}+\left|\partial_{\rho} W_{k}\right| \gamma_{\rho}\left(\left\|\xi_{1, r}\right\|\right) \\
& +\max \left\{1, \frac{1}{\rho_{k}}\right\}\left[\left|\beta_{k}\right| \gamma_{\beta}\left(\left\|\xi_{1, r}\right\|\right)+\left|\tilde{\alpha}_{k}\right| \gamma_{\alpha}\left(\left\|\xi_{1, r}\right\|\right)\right] .
\end{aligned}
$$

Assume, first, that $\max \left\{1,1 / \rho_{k}\right\}=1$. Then, let $\lambda_{1}, \lambda_{2}, \lambda_{3}>0$ be sufficiently large so that $c_{1}^{\prime}:=c_{1}-\frac{1}{2 \lambda_{1}}>0$, $c_{2}^{\prime}:=c_{2}-\frac{1}{2 \lambda_{3}}>0$, and $c_{3}^{\prime}:=\tilde{c}_{3}-\frac{1}{2 \lambda_{2}}>0$. In view of Young's inequality, from (25) we have

$$
\begin{aligned}
\dot{V}_{2, k}\left(\xi_{2, k}\right) \leq & -c_{1}^{\prime}\left[\partial_{\rho} W_{k}\right]^{2}-c_{2}^{\prime} \tilde{\alpha}_{k}^{2}-c_{3}^{\prime} \beta_{k}^{2}+\frac{\lambda_{1}}{2} \gamma_{\rho}\left(\left\|\xi_{1, r}\right\|\right)^{2} \\
& +\frac{\lambda_{2}}{2} \gamma_{\beta}\left(\left\|\xi_{1, r}\right\|\right)^{2}+\frac{\lambda_{3}}{2} \gamma_{\alpha}\left(\left\|\xi_{1, r}\right\|\right)^{2} \\
\leq & -c^{\prime}\left\|\xi_{2, k}\right\|^{2}+\gamma\left(\left\|\xi_{1, r}\right\|\right)
\end{aligned}
$$

where $\quad c^{\prime}:=\min \left\{c_{1}^{\prime}, c_{2}^{\prime}, c_{3}^{\prime}\right\}$ and $\gamma\left(\left\|\xi_{1, r}\right\|\right) \quad:=$ $\frac{\lambda_{1}}{2} \gamma_{\rho}\left(\left\|\xi_{1, r}\right\|\right)^{2}+\frac{\lambda_{2}}{2} \gamma_{\beta}\left(\left\|\xi_{1, r}\right\|\right)^{2}+\frac{\lambda_{3}}{2} \gamma_{\alpha}\left(\left\|\xi_{1, r}\right\|\right)^{2}$.

Now, let $\max \left\{1,1 / \rho_{k}\right\}=1 / \rho_{k}$. For any $\delta>0$ and for any $\rho_{k} \geq \delta$, we have $\max \left\{1,1 / \rho_{k}\right\} \leq 1 / \delta$. Then, define $\lambda_{4}, \lambda_{5}, \lambda_{6}>0$ such that $c_{1}^{\prime \prime}:=c_{1}-\frac{1}{2 \lambda_{4} \delta}>0$, $c_{2}^{\prime \prime}:=c_{2}-\frac{1}{2 \lambda_{6} \delta}>0$, and $c_{3}^{\prime \prime}:=\tilde{c}_{3}-\frac{1}{2 \lambda_{5} \delta}>0$. Applying Young's inequality on (25), we obtain

$$
\dot{V}_{2, k}\left(\xi_{2, k}\right) \leq-c^{\prime \prime}\left\|\xi_{2, k}\right\|^{2}+\frac{1}{\delta} \gamma\left(\left\|\xi_{1, r}\right\|\right)
$$

where, $c^{\prime \prime}:=\min \left\{c_{1}^{\prime \prime}, c_{2}^{\prime \prime}, c_{3}^{\prime \prime}\right\}$. Therefore, from (26) and (27), we see that the function $V_{p}$ in (22) with $p=2$, satisfies

$$
\dot{V}_{2}\left(\xi_{2}\right) \leq-\bar{c}\left\|\xi_{2}\right\|^{2}+\bar{\gamma}\left(\left\|\xi_{1}\right\|\right)
$$

where $\bar{\gamma}\left(\left\|\xi_{1}\right\|\right):=\max \left\{1, \frac{1}{\delta}\right\} \sum_{r=1}^{l_{1}} \gamma\left(\left\|\xi_{1, r}\right\|\right)$ and $\bar{c}=$ $\min \left\{c^{\prime}, c^{\prime \prime}\right\}$. Assume now that the solutions $t \mapsto \xi_{2}(t)$ grow unboundedly. Since $\xi_{1}(t)$ is bounded, there exists $t^{\prime}$ such that $\dot{V}_{2}\left(\xi_{2}(t)\right) \leq 0$ for all $t \geq t^{\prime}$, which leads to a contradiction. That is, $\xi_{2}(t)$ is bounded. Furthermore, since $\xi_{1}(t) \rightarrow 0$ it follows that, also, $\xi_{2}(t) \rightarrow 0$. The previous arguments hold for $p=3$ and, by induction, for any $p \leq h$.

So far we assumed that $\rho_{k}(t) \in \mathcal{J}$ for all $t \geq 0$. We show next that this holds under the Proposition's assumption that $\rho_{k}(0) \in \mathcal{J} \backslash\{0\}$. We proceed by contradiction. Suppose that there exists $T>0$ such that for all $t \in[0, T)$ and at least one $k \leq m, \rho_{k}(t) \in \mathcal{J}$ and $\rho_{k}(T) \notin \mathcal{J}$. Then, from the definition of the barrier functions $W_{k}$, we have that $W_{k}\left(\rho_{k}(t)\right) \rightarrow \infty$. This, however, contradicts (23) and (28), which imply that $W_{k}\left(\rho_{k}(t)\right)$ is bounded for all $t \geq 0$ and all $k \leq m$. Connectivity maintenance (equivalently, forward invariance of $\mathcal{J}$ ) follows.

\section{EXPERIMENTAL RESULTS}

To illustrate our formal results, some experiments were performed with four Nexter Robotics' Wifibots and the Robot Operating System (ROS) interface were used for the implementation of the control law. An Optitrack motion capture system based on active IR cameras and markers was used which, coupled with odometry sensors, allowed us to obtain positions and velocities of the robots for analysis purposes.

For the tests we consider a leader-follower full-consensusbased formation scenario, rather than a generic consensus one, in order to prevent collisions between the agents when close to the consensus point. It is assumed that the four mobile robots interact through a directed spanning tree as illustrated in Figure 3.

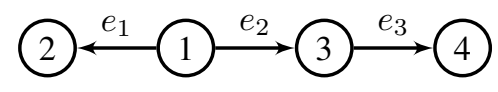

Fig. 3. Directed-tree topology used in experiments.

The leader is static due to space limitations in the test facility. The results are depicted in Figures 4-8 for initial conditions $\left(x_{1}(0), y_{1}(0), \theta_{1}(0)\right)=(1.5,2.2,0),\left(x_{2}(0), y_{2}(0), \theta_{2}(0)\right)=$ $(-1.3,2.2,-1.9),\left(x_{3}(0), y_{3}(0), \theta_{3}(0)\right)=(-1.5,-0.5,1.6)$, and $\left(x_{4}(0), y_{4}(0), \theta_{4}(0)\right)=(1.2,0.3,-2.8)$. In the testscenario each robot was subject to a limited communication range, hence imposing distance-based connectivity constraints. These constraints are encoded by the barrier function (10) as $U_{k}\left(\rho_{k}\right):=\ln \left(\frac{\Delta_{k}^{2}}{\Delta_{k}^{2}-\rho_{k}^{2}}\right)$, where the radii of the communication ranges were set to $\Delta_{1}=2.7 \mathrm{~m}, \Delta_{2}=3.4 \mathrm{~m}$, and $\Delta_{3}=4.2 \mathrm{~m}$.

In Figure 4 is presented the motion of the vehicles. The blue dot represents the position of the static leader and the red dots are the desired positions of each agent with respect to its respective leader as defined by the interaction graph showed in Figure 3. It is clear from Figures 5 and 6 that the group of agents successfully achieve the desired formation with consensus on the orientation. Additionally, in Figure 6 it can be appreciated that all distance constraints (dashed lines) are respected, thus guaranteeing connectivity maintenance.

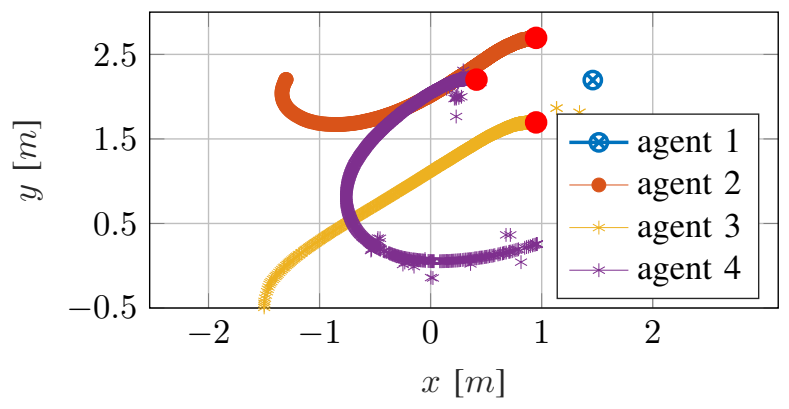

Fig. 4. Motion of the vehicles.

In Figures 7 and 8 are presented the control inputs for the four agents of the system. It is evident from the figures that the control inputs are smooth, as claimed. We clarify that the apparent "dripping" in the curves on Figures 7-8 is due to position-measurements noise coming from the motion capture system of our experimental benchmark. The effect of such noise can also be appreciated in Figures 4 and 6 . 


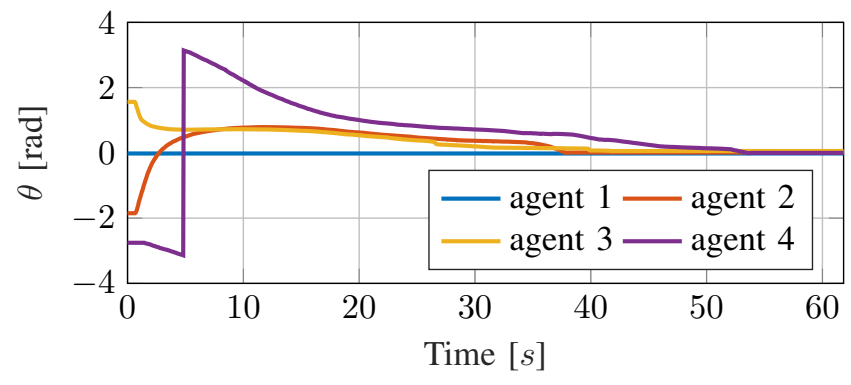

Fig. 5. Trajectories of the orientation of the vehicles.

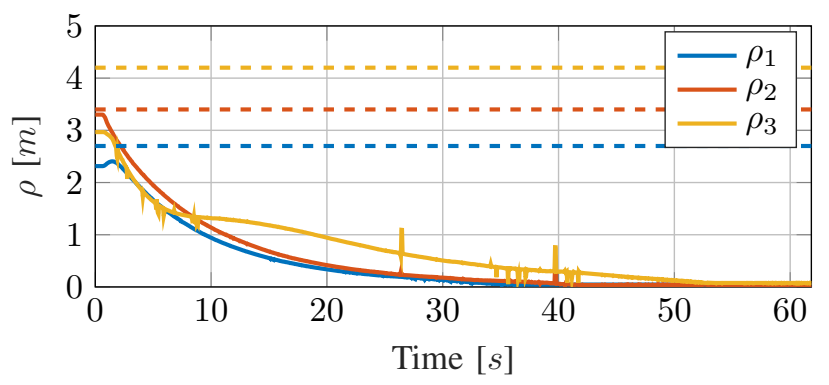

Fig. 6. Trajectories of the distance between neighbouring agents.

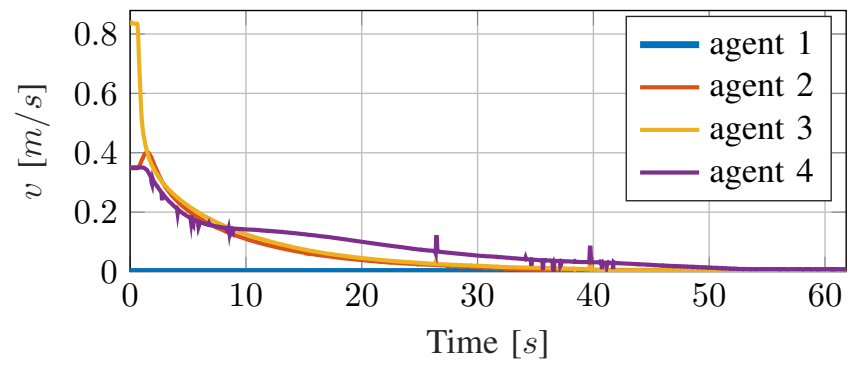

Fig. 7. Linear velocities.

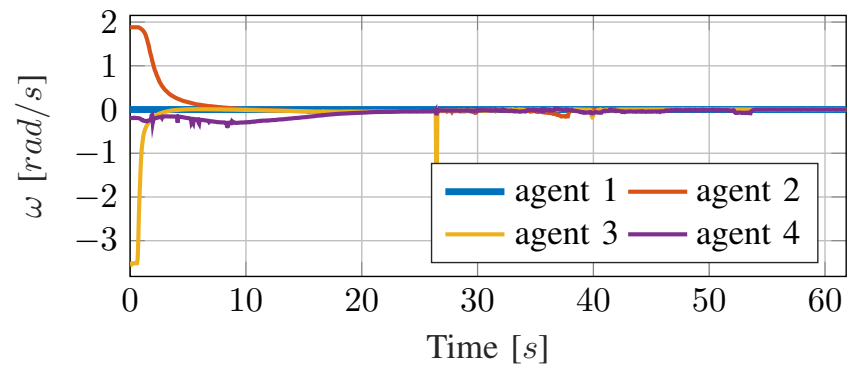

Fig. 8. Angular rates.

\section{CONCLUSIONS}

In this paper we presented a smooth time-invariant distributed feedback control law that solves the full consensus problem for swarms of unicycles with communication range constraints and interconnected through a directed-spanningtree communication topology. The control methodology is based on barrier Lyapunov functions and a polar-coordinatesbased model. The latter allows the use of smooth timeinvariant control laws and transforms the consensus problem into a stabilization one so that it may be analysed through classical Lyapunov theory. Furthermore, the proposed con- troller uses only relative information, making it well suited for practical applications.

Current and future work focuses on more general digraph topologies, higher-order systems, obstacle avoidance, and 3D autonomous vehicles.

\section{REFERENCES}

[1] J. Zhao and G.-P. Liu, "Time-Variant Consensus Tracking Control for Networked Planar Multi-Agent Systems with Non-Holonomic Constraints," J Syst Sci Complex, vol. 31, no. 2, pp. 396-418, Apr. 2018.

[2] K.-C. Cao, B. Jiang, and D. Yue, "Rendezvous of multiple nonholonomic unicycles-based on backstepping," International Journal of Control, vol. 91, no. 6, pp. 1271-1283, Jun. 2018.

[3] M. Maghenem, A. Loría, and E. Panteley, "Lyapunov-based formationtracking control of nonholonomic systems under persistency of excitation," IFAC-PapersOnLine, vol. 49, no. 18, pp. 404-409, 2016.

[4] M. Maghenem, A. Bautista, E. Nuno, A. Loría, and E. Panteley, "Consensus of multiagent systems with nonholonomic restrictions via Lyapunov's direct method," IEEE Control Syst. Lett., vol. 3, no. 2, pp. 344-349, Apr. 2019.

[5] A. Bautista-Castillo, C. Lopez-Franco, and E. Nuno, "Consensus-based formation control for multiple nonholonomic robots," in 2016 IEEE International Autumn Meeting on Power, Electronics and Computing (ROPEC), Ixtapa, Zihuatanejo, Mexico, Nov. 2016, pp. 1-6.

[6] D. Dimarogonas and K. Kyriakopoulos, "On the rendezvous problem for multiple nonholonomic agents," IEEE Transactions Automatic Control, vol. 52, no. 5, pp. 916-922, May 2007.

[7] Z. Kan, J. Klotz, T. Cheng, and W. E. Dixon, "Ensuring network connectivity for nonholonomic robots during decentralized rendezvous," in 2012 American Control Conference (ACC), 2012, pp. 3718-3723.

[8] Z. Kan, J. R. Klotz, J. M. Shea, E. A. Doucette, and W. E. Dixon, "Decentralized Rendezvous of Nonholonomic Robots With Sensing and Connectivity Constraints," Journal of Dynamic Systems, Measurement, and Control, vol. 139, no. 2, 112016.

[9] A. Ajorlou and A. G. Aghdam, "Connectivity preservation in nonholonomic multi-agent systems: A bounded distributed control strategy," IEEE Transactions on Automat. Contr., vol. 58, no. 9, pp. 2366-2371, 2013.

[10] J. Lian, Y. Meng, and L. Li, "Formation Control and Obstacle Avoidance for Multi-Agent Systems Using Barrier Lyapunov Functions," in 2018 Eighth International Conference on Information Science and Technology (ICIST), 2018, pp. 15-20.

[11] D. Panagou, D. M. Stipanovic, and P. G. Voulgaris, "Distributed coordination control for multi-robot networks using Lyapunov-like barrier functions," IEEE Transactions on Automat. Contr., vol. 61, no. 3, pp. 617-632, Mar. 2016.

[12] R. K. Williams, A. Gasparri, G. S. Sukhatme, and G. Ulivi, "Global connectivity control for spatially interacting multi-robot systems with unicycle kinematics," in 2015 IEEE International Conference on Robotics and Automation (ICRA), 2015, pp. 1255-1261.

[13] H. Poonawala, A. C. Satici, H. Eckert, and M. W. Spong, "Collisionfree formation control with decentralized connectivity preservation for nonholonomic-wheeled mobile robots," IEEE Transactions on Control of Network Systems, vol. 2, pp. 122-130, 2015.

[14] E. Restrepo, I. Sarras, A. Loría, and J. Marzat, "Leader-follower consensus of unicycle-type vehicles via smooth time-invariant feedback," in 2020 European Control Conference (ECC), Saint Petersburg, 2020.

[15] M. Aicardi, G. Casalino, A. Bicchi, and A. Balestrino, "Closed loop steering of unicycle like vehicles via Lyapunov techniques," IEEE Robotics and Automation Magazine, vol. 2, no. 1, pp. 27-35, Mar. 1995.

[16] Z. Lin, B. Francis, and M. Maggiore, "Necessary and sufficient graphical conditions for formation control of unicycles," IEEE Transactions on Automat. Contr., vol. 50, no. 1, pp. 121-127, 2005.

[17] M. Maghenem and A. Loría, "Lyapunov functions for persistentlyexcited cascaded time-varying systems: application in consensus analysis," IEEE Transactions on Automatic Control, vol. 62, no. 7, pp. 34163422, 2017.

[18] K. P. Tee, S. S. Ge, and E. H. Tay, "Barrier Lyapunov functions for the control of output-constrained nonlinear systems," Automatica, vol. 45, no. 4, pp. 918-927, Apr. 2009.

[19] E. Panteley and A. Loría, "Growth rate conditions for uniform asymptotic stability of cascaded time-varying systems," Automatica, vol. 37, no. 3, pp. 453-460, Mar. 2001. 